\title{
SCHREIER SYSTEMS IN FREE PRODUCTS
}

\author{
by I. M. S. DEY \\ (Received 18 June, 1964)
}

1. Introduction. In 1927 Schreier [8] proved the Nielsen-Schreier Theorem that a subgroup $H$ of a free group $F$ is a free group by selecting a left transversal for $H$ in $F$ possessing a certain cancellation property. Hall and Rado [5] call a subset $T$ of a free group $F$ a Schreier system in $F$ if it possesses this cancellation property, and consider the existence of a subgroup $H$ of $F$ such that a given Schreier system $T$ is a left transversal for $H$ in $F$.

Now if $G=\prod^{*} A_{j}(j \in J)$ is the free product of groups $A_{j}$, the Kurosh Subgroup Theorem enables one to determine the structure of a subgroup $H$ of $G$. This theorem of Kurosh can be proved (see for example Maclane [7], Kuhn [6], Weir [9]) by selecting for each $j \in J$ a left transversal $S_{j}$ for $H$ in $G$, such that $S=\bigcup_{j \in J} S_{j}$ possesses a certain generalized cancellation property. We call such a subset $S$ a uniform Schreier system in $G$ and study questions analogous to those of Hall and Rado. The questions are made explicit in $\S 2$ and answered in $\$ 3,4,5$. Necessary and sufficient conditions for the existence of a subgroup $H$ of $G$ associated with an arbitrary uniform Schreier system $S$ are given by Theorems 3.11 and 4.7 .

In $\S 6$ the structure of a subgroup $H$ of $G$ is discussed in the light of the methods evolved here and a formula (6.11) for the number of subgroups of finite index in $G$ is derived. This is a generalization of that of Hall [3] for free groups. In $\S 7$ the methods are used to give an alternative proof that the free product of two residually finite groups is residually finite (Gruenberg [2]).

The contents of this paper formed part of a Ph.D. thesis and I would like to record my thanks to my supervisors Dr Hanna Neumann and Dr J. Wiegold for their help and encouragement during this period of study.

2. Uniform Schreier systems and admissible functions. Let $G=\prod^{*} A_{j}(j \in J)$ be the free product of groups $A_{j}$ for all $j \in J$, some well-ordered index set. The elements of $G$ can be uniquely represented as reduced words $g \in G, g=a_{1} a_{2} \ldots a_{t}$, in the syllables $a_{i} \in A_{a_{i}}\left(\alpha_{i} \in J\right.$, $i=1,2, \ldots, t)$, where $\alpha_{1} \neq \alpha_{i+1}$. The length of $g, \lambda(g)$, is to be the number of syllables occurring in this reduced expression for $g$. Let $I$ be some other well-ordered index set, and let $S=\left\{s_{i j}\right.$, for all $i \in I, j \in J\}$ be a subset of $G$; then we make the following definition.

(2.1) Definition. The set $S=\left\{s_{i j}\right\}$ is a uniform Schreier system in $G$ if the following conditions are satisfied:

(i) $s_{1 j}=1$, the identity of $G$, for all $j \in J$.

(ii) If $s_{i j}=a_{1} a_{2} \ldots a_{t} \neq 1, i \neq 1$, and $\alpha_{t}=j$, then there exists $a p \in I, p \neq i$, such that

$$
s_{p j}=a_{1} a_{2} \ldots a_{t-1}=s_{p \alpha_{t-1}} \cdot
$$

(iii) If $s_{i j}=a_{1} a_{2} \ldots a_{t} \neq 1$ and $\alpha_{t} \neq j$, then $s_{i j}=s_{i \alpha_{t}}$.

(iv) If $s_{i j}=s_{p q}$, then $i=p$. 
It is convenient to consider a uniform Schreier system $S$ as a matrix, where $s_{i j}$ is the element in the $(i, j)$ th place. We can then speak of the rows and columns of $S$. We denote the $j$ th column of $S$ by $S_{j}$, so that $S=\bigcup_{j \in J} S_{j}$. We shall also require the following sets $\tilde{S}_{j}$ and $S_{j}^{*}$, where $\tilde{S}_{j}=S \cup S_{j} A_{j}$ and $S_{j}^{*}$ is the subset of those elements of $S_{j}$ whose last syllable does not lie in $A_{j}$, together with $s_{1 j}$.

(2.2) We note the following easy consequence of the definition:

If $s_{i j} \in S$, where $S$ is a uniform Schreier system, and if $s_{i j}=a_{1} a_{2} \ldots a_{t}$, then

for $r=1,2, \ldots, t-1$.

$$
a_{1} a_{2} \ldots a_{r} \in S_{\alpha_{r}} \cap S_{\alpha_{r+1}}
$$

The following lemma ensures the existence of uniform Schreier systems.

(2.3) Lemma (Maclane). Let $G=\prod^{*} A_{j}(j \in J)$ be the free product of arbitrary groups $A_{j}$, and let $H$ be any subgroup of $G$. Then, for each $j \in J$, we can find a left transversal $S_{j}$ for $H$ in $G$, such that $S=\bigcup_{j \in J} S_{j}$ is a uniform Schreier system in $G$.

The proof can be found in Maclane [7]. This lemma not only shows generally that uniform Schreier systems exist but also shows that with each subgroup $H$ of $G$ one can associate at least one uniform Schreier system $S$. It is this association that is studied here.

Again, let $H$ be a subgroup of $G$, the free product of groups $A_{j}(j \in J)$; then, if $g_{1}, g_{2} \in G$ lie in the same left coset of $G$ modulo $H$, we shall write $g_{1} \sim g_{2}$ and $\sim$ is then an equivalence relation on $G$. Now, from (2.3), if $g \in G$, there is a uniform Schreier system $S$ for $H$ in $G$ such that $g \sim s_{i j} \in S$, for all $j \in J$ and some $i \in I$ depending on $g$. This permits us to define coset functions $\psi_{j}$ (e.g. Kuhn [6], Weir [9]) with arguments in $G$ and values in $S_{j}$.

(2.4) Definition. If $H$ is a subgroup of $G$ and $S$ is a uniform Schreier system for $H$ in $G$, then the coset functions $\psi_{j}$ for each $j \in J$ are defined as follows: if $g \in G, g \sim s_{i j} \in S_{j}(i \in I, j \in J)$, then $\psi_{j}(g)=s_{i j}$.

It follows from the proof of Lemma (2.3) that the coset functions defined on $G$ with values in $S$ as chosen there satisfy the condition

(2.5) $\psi_{j}(g a) \in \psi_{j}(g) A_{j}$, for all $a \in A_{j}, j \in J$.

We therefore make the definition:

(2.6) Definition. If $H$ is a subgroup of $G$ and $S$ is a uniform Schreier system for $H$ in $G$, then $H$ and $S$ will be said to be associated if the corresponding coset functions defined by (2.4) satisfy (2.5).

Lemma (2.3) states that each subgroup $H$ of $G$ can be associated with at least one uniform Schreier system $S$ in $G$ and it is natural to consider the converse question, namely, if $S$ is a uniform Schreier system in $G$, is there any subgroup $H$ of $G$ with which $S$ can be associated? To answer this question we examine the coset functions and introduce the concept of a set of admissible functions for a uniform Schreier system $S$ (cf. Hall and Rado [5]).

Let $S$ be a uniform Schreier system associated with a subgroup $H$ of $G$ and let $\phi_{j}$ be the restriction of $\psi_{j}$ to the set $\widetilde{S}_{j}=S \cup S_{j} A_{j}$; then we have 
(2.7) Lemma. Let $g \in G, g=a_{1} a_{2} \ldots a_{t}\left(a_{i} \in A_{\alpha_{i}}, \alpha_{i} \in J\right)$ and put

$$
h_{0}=\phi_{j}(1)=s_{1 j}, \quad h_{i}=\phi_{j}\left(\phi_{\alpha_{i}}\left(\phi_{\alpha_{i}}\left(h_{i-1}\right) a_{i}\right)\right)
$$

Then $h_{t}=\psi_{j}(g)$.

The proof follows from the properties of the equivalence relation $\sim$ and the definition of $\psi_{j}$

Hence to calculate $\psi_{j}(g)$ we only require the values of $\phi_{j}$ defined on $\tilde{S}_{j}$.

(2.8) Lemma. The functions $\phi_{j}$ defined on $\tilde{S}_{j}$ with values in $S_{j}$ satisfy the following conditions.

(i) $\phi_{j}\left(s_{i j} a\right)=s_{p j}$ for some $p \in I$, and all $a \in A_{j}$.

(ii) $\phi_{j}\left(s_{p q}\right)=s_{p j}$ for all $s_{p q} \in S$.

(iii) $\phi_{j}\left(s_{i j} a\right) \in s_{i j} A_{j}$ for all $a \in A_{j}$.

(iv) $\phi_{j}\left(\phi_{j}\left(s_{i j} a\right) a^{\prime}\right)=\phi_{j}\left(s_{i j} a a^{\prime}\right)$ for all $a, a^{\prime} \in A_{j}$.

(v) $\phi_{j}\left(\phi_{j}\left(s_{i j} a\right) a^{-1}\right)=s_{i j}$.

Proof. (i), (ii) follow from the definition of $\psi_{j}$, (iii) from the association of $H$ and $S$. (iv) follows from the fact that, if $\phi_{j}\left(s_{i j} a\right)=s_{p j}$, then $s_{i j} a \sim s_{p j}$ and $s_{i j} a a^{\prime} \sim s_{p j} a^{\prime}$. So

$$
\phi_{j}\left(s_{i j} a a^{\prime}\right)=\phi_{j}\left(s_{p j} a^{\prime}\right)=\phi_{j}\left(\phi_{j}\left(s_{i j} a\right) a^{\prime}\right) .
$$

(v) follows from (ii) and (iv) with $a^{\prime}=a^{-1}$.

Suppose that $S$ is a uniform Schreier system in $G$ and that for each $j \in J$ there is some function $\phi_{j}$ defined on $S_{j}$ with values in $S_{j}$. Put $\Phi=\left\{\phi_{j}: j \in J\right\}$; then we have

(2.9) Definition. $\Phi$ is a set of admissible functions for $S$ if the functions $\phi_{j}$ satisfy the conditions of (2.8).

Thus we have that, if $H$ is a subgroup of $G$ and $S$ an associated uniform Schreier system for $H$, we can construct a set of admissible functions $\Phi$ for $S$.

$S$ and $\Phi$ can now be used to describe the structure of $H$ (cf. Maclane [7]).

(2.10) THEOREM (Kurosh). Let $H$ be a subgroup of the free product $G, S$ an associated uniform Schreier system for $H$. If $\Phi$ is the set of admissible functions constructed for $S$, then

$$
H=F^{*} \prod_{j \in J}^{*}\left(\prod_{s_{i j} \in S_{j}^{*}}^{*} s_{i j} A_{j} s_{i j}^{-1} \cap H\right),
$$

where $S_{j}^{*}$ is the set of those elements of $S_{j}$ whose last syllable does not lie in $S_{j}$ together with the unit element. Further

(2.11) $F$ is a free group freely generated by the subset of $Q=\left\{s_{i j} s_{i q}^{-1}\right.$, for all $i \in I$ and all $j$, $q \in J\}$ obtained by deleting unit elements and possible repetitions from $Q$, and $\left.a \in A_{j}\right\}$.

(2.12) $s_{i j} A_{j} s_{i j}^{-1} \cap H$ is generated by the set of elements $Q_{i j}=\left\{s_{i j} a \phi_{j}\left(s_{i j} a\right)^{-1}\right.$, for all 
If a subgroup $H$ of $G$ is described in this way by some associated uniform Schreier system $S$ and a set of admissible functions $\Phi$, we shall write $H=\langle S, \Phi\rangle$ and call this a representation of $H$. We can now state a series of problems which will be discussed in the following sections. Let $H$ be a subgroup of $G, S$ a uniform Schreier system in $G$ and $\Phi$ a set of admissible functions for $S$.

(2.13) Given $S$, determine all the possible $H$ associated with $S$, if any.

(2.14) Given $S$, determine all the possible $\Phi$ for $S$, if any.

(2.15) Given $S$ and $\Phi$, determine all those $H$ such that $H=\langle S, \Phi\rangle$, if any.

(2.16) Given $H$, determine all the representations $H=\langle S, \Phi\rangle$.

We have seen that, if $S$ and $H$ are associated, we can construct a suitable $\Phi$; so the solution to problem (2.13) will follow from those to problems (2.14) and (2.15).

3. Problem (2.15). In this section we solve problem (2.15), that is, we assume that $S$ is a uniform Schreier system and that $\Phi$ is a set of admissible functions for $S$ and we determine all the subgroups $H$ with the representation $H=\langle S, \Phi\rangle$.

We start by considering a particular $\phi_{j} \in \Phi$, and use its properties (2.8) to define a function $\psi_{j}$ from $G$ to $S_{j}$.

(3.1) Definition. If $g \in G$, define $\psi_{j}(g)$ as follows:

(i) If $g=1, \psi_{j}(g)=s_{1 j}=1$.

(ii) If $g \neq 1, g=a_{1} a_{2} \ldots a_{t}$, where $a_{i} \in A_{\alpha_{i}}, \alpha_{i} \in J$, we put $h_{0}=s_{1 j}$, and

$$
h_{l}=\phi_{j}\left(\phi_{a_{i}}\left(\phi_{a_{i}}\left(h_{i-1}\right) a_{i}\right)\right)
$$

then $\psi_{j}(g)$ is to be $\psi_{j}(g)=h_{t}$.

This $\psi_{j}$ is clearly a well-defined mapping, since each $g \in G$ has a unique representation as a reduced word. We also see that, if $g_{i}=a_{1} a_{2} \ldots a_{i}$, then the nature of the constructive process implies that $\psi_{j}\left(g_{i}\right)=h_{i}$.

We now prove several lemmas describing the properties of this function $\psi_{j}$.

(3.2) LemmA. Let $g \in G, g=a_{1} a_{2} \ldots a_{t}\left(a_{i} \in A_{a_{i}}, i=1,2, \ldots, t\right)$ and let $a \in A_{\alpha}$ for some $\alpha \in I$. Then $\psi_{j}(g)$ is the same word as that obtained by applying the construction process of (3.1) to

$$
g^{\prime}=a_{1} a_{2} \ldots a_{i} a a^{-1} a_{i+1} \ldots a_{i} .
$$

Proof. Assume that in calculating $\psi_{j}(g)$ we obtain the sequence of terms $h_{0}, h_{1}, \ldots, h_{t}$, and that in applying the process to $g^{\prime}$ we obtain the sequence $h_{0}^{\prime}, h_{1}^{\prime}, \ldots, h_{t+2}^{\prime}$. Then, from a remark above, $h_{i}=\psi_{j}\left(a_{1} a_{2} \ldots a_{i}\right)=h_{i}^{\prime}$. Consider

$$
h_{i+1}^{\prime}=\phi_{j}\left(\phi_{\alpha}\left(\phi_{\alpha}\left(h_{i}^{\prime}\right) a\right)\right)=\phi_{j}\left(\phi_{\alpha}\left(\phi_{\alpha}\left(h_{i}\right) a\right)\right),
$$

and

$$
h_{i+2}^{\prime}=\phi_{j}\left(\phi_{\alpha}\left(\phi_{\alpha}\left(h_{i+1}^{\prime}\right) a^{-1}\right)\right)=\phi_{j}\left(\phi_{\alpha}\left(\phi_{\alpha}\left(\phi_{j}\left(\phi_{\alpha}\left(\phi_{\alpha}\left(h_{i}\right) a\right)\right)\right) a^{-1}\right)\right) \text {. }
$$


Now it is a simple consequence of $(2.8(\mathrm{ii}))$ that, if $s \in S, \phi_{j}(s)=\phi_{j}\left(\phi_{a}(s)\right)$; so, putting

we have

$$
\phi_{\alpha}\left(\phi_{\alpha}\left(h_{i}\right) a\right)=s \in S_{\alpha},
$$

$$
\begin{aligned}
h_{i+2}^{\prime} & =\phi_{j}\left(\phi_{\alpha}\left(\phi_{\alpha}\left(\phi_{j}(s)\right) a^{-1}\right)\right) \\
& =\phi_{j}\left(\phi_{\alpha}\left(\phi_{\alpha}(s) a^{-1}\right)\right) \\
& =\phi_{j}\left(\phi_{\alpha}\left(s a^{-1}\right)\right), \text { by }(2.8(\mathrm{ii})), \\
& =\phi_{j}\left(\phi_{\alpha}\left(\phi_{\alpha}\left(\phi_{\alpha}\left(h_{i}\right) a\right)^{-1} a\right)\right) .
\end{aligned}
$$

But, by $(2.8(v))$, this implies that

$$
h_{i+2}^{\prime}=\phi_{j}\left(\phi_{\alpha}\left(\phi_{\alpha}\left(h_{i}\right)\right)\right)=\phi_{j}\left(h_{i}\right)=h_{i} .
$$

We see, therefore, that on completing the construction process, $h_{t}=h_{t+2}^{\prime}$, proving the lemma.

This lemma implies that, if $g, g^{\prime} \in G$, then in calculating $\psi_{j}\left(g g^{\prime}\right)$ we do not have to consider any possible cancellations between $g$ and $g^{\prime}$.

(3.3) Lemma. The restriction of $\psi_{j}$ to $\tilde{S}_{j}$ is $\phi_{j}$.

Proof. 1. We first show that, if $s \in S$, then $\psi_{j}(s)=\phi_{j}(s)=s$.

(i) If $s \in S$ and $s=1$, then, from (3.1), $\psi_{j}(s)=1=\phi_{j}(s)$.

(ii) Suppose now that $s \in S, s \neq 1$, and that $s=a_{1} a_{2} \ldots a_{t}\left(a_{i} \in A_{\alpha_{1}}\right)$. We put $h_{0}=1=s_{1}$, and calculate $h_{1}$.

$$
h_{1}=\phi_{j}\left(\phi_{a_{1}}\left(\phi_{a_{1}}\left(h_{0}\right) a_{1}\right)\right)=\phi_{j}\left(\phi_{a_{1}}\left(a_{1}\right)\right) \text {. }
$$

Now, from (2.2), $a_{1} \in S_{\alpha_{1}} \cap S_{a_{2}}$; so $\phi_{\alpha_{1}}\left(a_{1}\right)=a_{1}=\phi_{\alpha_{2}}\left(a_{1}\right)$, and $h_{1}=\phi_{j}\left(a_{1}\right)$. Again, from the construction,

$$
\left.h_{2}=\phi_{j}\left(\phi_{\alpha_{2}}\left(h_{1}\right) a_{2}\right)\right)=\phi_{j}\left(\phi_{a_{2}}\left(\phi_{a_{2}}\left(\phi_{j}\left(a_{1}\right)\right) a_{2}\right)\right) .
$$

But, from a remark in the proof of (3.2) and from (2.2),

$$
\phi_{a_{2}}\left(\phi_{j}\left(a_{1}\right)\right)=\phi_{a_{2}}\left(a_{1}\right)=a_{1}
$$

so $h_{2}=\phi_{j}\left(\phi_{a_{2}}\left(a_{1} a_{2}\right)\right)$. But, again from (2.2), $a_{1} a_{2} \in S_{\alpha_{2}} \cap S_{\alpha_{3}}$; so $\phi_{\alpha_{2}}\left(a_{1} a_{2}\right)=a_{1} a_{2}=\phi_{\alpha_{3}}\left(a_{1} a_{2}\right)$, and $h_{2}=\phi_{j}\left(a_{1} a_{2}\right)$. We repeat this argument to show that

$$
\phi_{j}(s)=h_{t}=\phi_{j}\left(a_{1} a_{2} \ldots a_{t}\right)=\psi_{j}(s) .
$$

2. Finally, we have to show that, if $s a \in S_{j} A_{j}$, then $\psi_{j}(s a)=\phi_{j}(s a)$.

(i) Again, if $s a=1, \psi_{j}(s a)=\phi_{j}(s a)$.

(ii) Now, if $s a \neq 1$, from (3.2), we do not have to consider any cancellations which might occur; so assume that, in calculating $\psi_{j}(s a)$, we obtain a sequence of terms $h_{0}, h_{1}, \ldots h_{t}$, where $h_{t-1}=\psi_{j}(s)=\phi_{j}(s)$, from the first part. Now consider $h_{t}$. We have $h_{t}=\phi_{j}\left(\phi_{j}\left(\phi_{j}(s) a\right)\right)$, where $\phi_{j}(s)=s$, since $s \in S_{j}$. So $h_{\mathrm{t}}=\phi_{j}\left(\phi_{j}(s a)\right)=\phi_{j}(s a)$. That is, $\psi_{j}(s a)=\phi_{j}(s a)$.

We have used in these proofs a simple consequence of (2.8(ii)), namely that, if $s \in S$, then $\phi_{j}(s)=\phi_{j}\left(\phi_{k}(s)\right)$, and from the previous lemma we immediately have $\psi_{j}(s)=\psi_{j}\left(\psi_{k}(s)\right)$. We extend this property to the whole of $G$. 
(3.4) Lemma. If $g \in G$, then $\psi_{j}(g)=\psi_{j}\left(\psi_{k}(g)\right)$.

Proof. We assume that, in calculating $\psi_{j}(g), \psi_{k}(g)$, we obtain the sequences $h_{0}, h_{1}, \ldots, h_{t}$ and $h_{0}^{\prime}, h_{1}^{\prime}, \ldots, h_{t}^{\prime}$, respectively. Then, clearly, since $h_{0}=s_{1 j}=1$, and $h_{0}^{\prime}=s_{1 k}=1, \phi_{j}\left(h_{0}^{\prime}\right)$ $=h_{0}$. We make the inductive hypothesis that $\phi_{j}\left(h_{i-1}^{\prime}\right)=h_{i-1}$; then

$$
h_{i}=\phi_{j}\left(\phi_{\alpha_{i}}\left(\phi_{a_{i}}\left(h_{i-1}\right) a_{i}\right)\right)=\phi_{j}\left(\phi_{\alpha_{i}}\left(\phi_{\alpha_{i}}\left(\phi_{j}\left(h_{i-1}^{\prime}\right)\right) a_{i}\right)\right) \text {. }
$$

Now, since $\phi_{\alpha_{i}} \phi_{j}=\phi_{\alpha_{i}}$ on $S$,

$$
h_{i}=\phi_{j}\left(\phi_{\alpha_{i}}\left(\phi_{\alpha_{i}}\left(h_{i-1}^{\prime}\right) a_{i}\right)\right)
$$

Because $\phi_{\alpha_{i}}\left(\phi_{\alpha_{i}}\left(h_{i-1}^{\prime}\right) a_{i}\right) \in S$,

$$
\left.h_{i}=\phi_{j}\left(\phi_{k}\left(\phi_{a_{i}}\left(h_{i-1}^{\prime}\right) a_{i}\right)\right)\right)=\phi_{j}\left(h_{i}^{\prime}\right) .
$$

Putting $i=t$, we have $\psi_{j}(g)=\phi_{j}\left(\psi_{k}(g)\right)$, and, from (3.3),

$$
\psi_{j}(g)=\psi_{j}\left(\psi_{k}(g)\right)
$$

We can now prove a most important lemma.

(3.5) Lemma. If $g, g^{\prime} \in G$, then $\psi_{j}\left(g g^{\prime}\right)=\psi_{j}\left(\psi_{j}(g) g^{\prime}\right)$.

Proof. We prove this lemma in three stages.

(3.6) If $s_{i q} \in S_{q}$, and $a \in A_{j}$, then $\psi_{j}\left(s_{i q} a\right)=\psi_{j}\left(s_{i j} a\right)$.

Proof of (3.6). Suppose that in calculating $\psi_{j}\left(s_{i q} a\right)$ we obtain a sequence of terms $h_{0}, h_{1}, \ldots, h_{t} ;$ then

We calculate $h_{t}$

$$
h_{t-1}=\psi_{j}\left(s_{i q}\right)=\phi_{j}\left(s_{i q}\right)=s_{i j}
$$

$$
h_{t}=\phi_{j}\left(\phi_{j}\left(\phi_{j}\left(s_{i j}\right) a\right)\right)=\phi_{j}\left(s_{i j} a\right) .
$$

That is, from (3.3), $\psi_{j}\left(s_{i q} a\right)=h_{t}=\psi_{j}\left(s_{i j} a\right)$

(3.7) If $g \in G$ and $a \in A_{\alpha}$ for some $\alpha \in J$, then

$$
\psi_{j}(g a)=\psi_{j}\left(\psi_{j}(g) a\right) .
$$

Proof of (3.7). Again we suppose that in calculating $\psi_{j}(g a)$ we obtain a sequence $h_{0}, h_{1}, \ldots, h_{t}$, where $h_{t-1}=\psi_{j}(g)$. We calculate $h_{t}$.

$$
h_{t}=\phi_{j}\left(\phi_{\alpha}\left(\phi_{\alpha}\left(h_{t-1}\right) a\right)\right) \text {. }
$$

Suppose that $h_{t-1}=s_{i j}$; then, from (2.8(ii)) and (3.3),

$$
h_{i}=\psi_{j}\left(\psi_{\alpha}\left(s_{i \alpha} a\right)\right) \text {, }
$$

and, from (3.6), this is $h_{t}=\psi_{j}\left(\psi_{\alpha}\left(s_{i j} a\right)\right)$. That is, from (3.4),

$$
\psi_{j}(g a)=\psi_{j}\left(s_{i j} a\right)=\psi_{j}\left(h_{t-1} a\right)=\psi_{j}\left(\psi_{j}(g) a\right) .
$$

We now return to the proof of (3.5). 
Proof of (3.5). We use an induction argument over the length of $g^{\prime}$.

(i) If $\lambda\left(g^{\prime}\right)=0$, then $g^{\prime}=1$ and $\psi_{j}\left(g g^{\prime}\right)=\psi_{j}\left(\psi_{j}(g) g^{\prime}\right)$.

(ii) If $\lambda\left(g^{\prime}\right)=1$, then $g^{\prime} \in A_{j}$ for some $j \in J$, and this case is covered by (3.7).

(iii) Now let $\lambda\left(g^{\prime}\right)>1$, and write $g^{\prime}=g^{\prime \prime} a$, where $a \in A_{\alpha}$ for some $\alpha \in J$. Then, by the inductive hypothesis,

Hence

$$
\psi_{j}\left(g g^{\prime \prime}\right)=\psi_{j}\left(\psi_{j}(g) g^{\prime \prime}\right)
$$

$$
\begin{aligned}
\psi_{j}\left(g g^{\prime}\right) & =\psi_{j}\left(g g^{\prime \prime} a\right) \\
& =\psi_{j}\left(\psi_{j}\left(g g^{\prime \prime}\right) a\right), \quad \text { from }(3.7), \\
& =\psi_{j}\left(\psi_{j}\left(\psi_{j}(g) g^{\prime \prime}\right) a\right), \text { by hypothesis, } \\
& =\psi_{j}\left(\psi_{j}(g) g^{\prime \prime} a\right), \quad \text { from }(3.7), \\
& =\psi_{j}\left(\psi_{j}(g) g^{\prime}\right) .
\end{aligned}
$$

This completes the proof of the lemma.

We now have the properties of $\psi_{j}$ required to solve problem (2.15). We start by defining an equivalence relation on $G$.

(3.8) Definition. If $g, g^{\prime} \in G$, we say that $g$ and $g^{\prime}$ are equivalent if there is a $j \in J$ such that $\psi_{j}(g)=\psi_{j}\left(g^{\prime}\right)$.

This is clearly an equivalence relation, and it is independent of $j$, because if $k \in J, k \neq j$, from (3.4),

$$
\psi_{k}(g)=\psi_{k}\left(\psi_{j}(g)\right)=\psi_{k}\left(\psi_{j}\left(g^{\prime}\right)\right)=\psi_{k}\left(g^{\prime}\right)
$$

Also, from the definition of the $\psi_{j}$, each $g \in G$ is equivalent to some $s_{i j} \in S_{j}$ and, since no two elements of $S_{j}$ are equal, we can take the elements of $S_{j}$ as representatives of the equivalence classes. That is, $G$ is partitioned into disjoint subsets indexed by the elements of $I$. We can now prove the following lemma.

(3.9) LEMma. The equivalence class containing $1 \in G$, is a subgroup $H$ of $G$.

Proof. (i) From the definition of $H, 1 \in H$.

(ii) Let $g, g^{\prime} \in H$; then $\psi_{j}(g)=\psi_{j}\left(g^{\prime}\right)=1$. But, from (3.5),

$$
\psi_{j}\left(g g^{\prime}\right)=\psi_{j}\left(\psi_{j}(g) g^{\prime}\right)=\psi_{j}\left(g^{\prime}\right)=1 \text {; }
$$

so $g g^{\prime} \in H$.

(iii) Let $g \in H$; then $\psi_{j}(g)=1$. Again from (3.5),

$$
\psi_{j}\left(g g^{-1}\right)=\psi_{j}\left(\psi_{j}(g) g^{-1}\right)=\psi_{j}\left(g^{-1}\right) .
$$

But $g g^{-1}=1 \in H$; so $\psi_{j}\left(g g^{-1}\right)=1=\psi_{j}\left(g^{-1}\right)$, and $g^{-1} \in H$.

This subgroup is to be the one with the representation $H=\langle S, \Phi\rangle$. We first prove

(3.10) Lemma. $S_{j}$ is a left transversal for $H$ in $G$. 
Proof. We denote the equivalence class containing $s_{i j} \in S_{j}$ by $H_{i}$, so that $H=H_{1}$; then we show that $H s_{i j}=H_{i}$.

1. Let $g \in G$, and consider the left coset $H g$. A typical element is $h g \in H g$, and we calculate $\psi_{j}(h g)$. From (3.5),

$$
\begin{aligned}
\psi_{j}(h g) & =\psi_{j}\left(\psi_{j}(h) g\right) \\
& =\psi_{j}(g), \quad \text { since } h \in H, \\
& =s_{i j}, \quad \text { say. }
\end{aligned}
$$

That is, $H g \in H_{i}$, and so each equivalence class contains at least one left coset, $H s_{i j}$.

2. Now let $H g, H g^{\prime}$ lie in $H_{i}$; then $g, g^{\prime} \in H_{i}$, and $\psi_{j}(g)=\psi_{j}\left(g^{\prime}\right)$. Now calculate $\psi_{j}\left(g^{\prime} g^{-1}\right)$ using (3.5).

$$
\psi_{j}\left(g^{\prime} g^{-1}\right)=\psi_{j}\left(\psi_{j}\left(g^{\prime}\right) g^{-1}\right)=\psi_{j}\left(\psi_{j}(g) g^{-1}\right)=\psi_{j}\left(g g^{-1}\right)=1 .
$$

That is, $g^{\prime} g^{-1} \in H$, and so $H g=H g^{\prime}$. This completes the proof of the lemma.

We have now shown that the left cosets of $H$ in $G$ are indexed by $I$, and that each $S_{j}$ is a left transversal for $H$ in $G$. Clearly the coset functions (2.4), which can now be defined, are, in fact, the $\psi_{j}$; so $H$ and $S$ are associated, and $\Phi$ is a set of admissible functions for $S$. That is, $H$ has the representation $H=\langle S, \Phi\rangle$.

Now clearly, if $H^{\prime}=\langle S, \Phi\rangle$ is another subgroup with the same representation as $H$, then, from (2.10), both have precisely the same free decomposition, so that $H=H^{\prime}$.

We can summarize this section in a theorem which states the solution to problem (2.15).

(3.11) THEOREM. If, in a free product $G$, we are given a uniform Schreier system $S$ and $a$ set $\Phi$ of admissible functions for $S$, then there is precisely one subgroup $H$ of $G$ such that $H$ has the representation

$$
H=\langle S, \Phi\rangle \text {. }
$$

4. Problem (2.14). In this section we solve problem (2.14), namely, given a uniform Schreier system $S$, determine all the sets of admissible functions $\Phi$, if any, defined on $S$. This requires that, for each $j \in J$, we have to define a function $\phi_{j}$ from $\tilde{S}_{j}$ to $S_{j}$ satisfying the conditions of (2.8). The conditions (2.8(ii)) determine precisely the values of the $\phi_{j}$ at elements of $S$. The only possibility of any choice is in defining the $\phi_{j}$ on $S_{j} A_{j}$ to satisfy (2.8(iii), (iv)). We consider the possible choices by looking at the effect of a $\phi_{j} \in \Phi$, a given set of admissible functions for $S$. This function $\phi_{j}$ can be considered as a matrix:

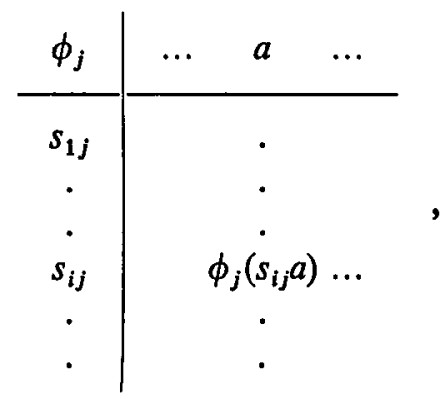


where the rows are indexed by the elements of $S_{j}$, and the columns by the elements $a \in A_{j}$. The element in the row $s_{i j}$ and the column $a$, is to be $\phi_{j}\left(s_{i j} a\right)$. We prove the following lemma.

(4.2) LeMMA. Each column of the matrix (4.1) is a permutation of $S_{j}$.

Proof. We consider the column indexed by $a \in A_{j}$.

(i) Since $\phi_{j}\left(s_{i j} a\right) \in S_{j}$, each element in the column is in $S_{j}$.

(ii) If $s_{i j} \in S_{j}$, then $\phi_{j}\left(s_{i j} a^{-1}\right)=s_{k j}$ occurs in the column headed by $a^{-1}$; so, in the column headed by $a$,

$$
\phi_{j}\left(s_{k j} a\right)=\phi_{j}\left(\phi_{j}\left(s_{i j} a^{-1}\right) a\right)=s_{i j},
$$

since $\phi_{j}$ satisfies (2.8(iv)). That is, each $s_{i j} \in S_{j}$ occurs in each column.

(iii) Suppose that $\phi_{j}\left(s_{i j} a\right)=\phi_{j}\left(s_{k j} a\right)$; then

$$
\phi_{j}\left(\phi_{j}\left(s_{i j} a\right) a^{-1}\right)=\phi_{j}\left(\phi_{j}\left(s_{k j} a\right) a^{-1}\right),
$$

and $s_{i j}=s_{k j}$. But, from (1.1(iv)), this implies that $i=k$; so each $s_{i j}$ occurs only once in the column. This completes the proof.

We use this lemma to define a mapping of $A_{j}$ into the set of permutations of the index set $I$.

(4.3) Definition. Let $a \in A_{j}, i \in I$; then $\pi_{j}(a)$ is to be the permutation corresponding to $a$, such that $i \pi_{j}(a)=p$ if and only if $\phi_{j}\left(s_{i j} a\right)=s_{p j}$.

We form the set $\Pi\left(A_{j}\right)=\left\{\pi_{j}(a)\right.$, for all $\left.a \in A_{j}\right\}$.

(4.4) Lemma. $\Pi\left(A_{j}\right)$ is a permutation representation of $A_{j}$ on the set $I$.

Proof. We only have to show that, if $a, a^{\prime} \in A_{j}$, then $\pi_{j}\left(a a^{\prime}\right)=\pi_{j}(a) \pi_{j}\left(a^{\prime}\right)$. Now if $i \in I$, consider $s_{i j}$; then, from (2.8(iv)),

that is,

$$
\phi_{j}\left(\phi_{j}\left(s_{i j} a\right) a^{\prime}\right)=\phi_{j}\left(s_{i j} a a^{\prime}\right),
$$

$$
i \pi_{j}\left(a a^{\prime}\right)=i\left(\pi_{j}(a) \pi_{j}\left(a^{\prime}\right)\right),
$$

for all $i \in I$, so that $\pi_{j}\left(a a^{\prime}\right)=\pi_{j}(a) \pi_{j}\left(a^{\prime}\right)$.

Much of the remaining work in this and the following sections is a study of these permutation representations $\Pi\left(A_{j}\right)$. We start by determining the transitivity classes of $\Pi\left(A_{j}\right)$, and for this we introduce some more terminology.

The elements of $S_{j}^{*}$ form a subset of $S_{j}$, which will be denoted by $S_{j}^{*}=\left\{s_{i_{j}}\right\}$, so that, if $s_{i j} \in S_{j}$, then, from (1.1(ii)), $s_{i j}=s_{i, j} a$, for some $r$, and for some $a \in A_{j}$. We use this fact to split $S_{j}$ into non-empty disjoint subsets $S_{i_{r} j}$, one to each $s_{i_{r} j} \in S_{j}^{*}$, where $s_{i j} \in S_{i_{r} j}$ if there is an $a \in A_{j}$ such that $s_{i j}=s_{i_{r} j} a$.

Clearly, each $S_{i_{r} j}$ contains precisely one of the $s_{i_{r} j}$. We can now describe a similar decomposition of $I$ into non-empty disjoint subsets $I_{r j}$.

(4.5) Definition. $I_{r j}$ is to be the subset of I such that $i \in I_{r j}$ if and only if $s_{i j} \in S_{i_{r j}}$. We shall call the $I_{r j}$ the blocks of $I$.

We can immediately prove 
(4.6) LEMma. The blocks $I_{r j}$ of $I$ are the transitivity classes of $\Pi\left(A_{j}\right)$.

Proof. Let $i \in I$ be one of the indices such that $s_{i j}=s_{i_{r} j} \in S_{j}^{*}$, and let $p$ be some other index in the block containing $i$; then there exists an $a \in A_{j}$ such that $s_{p j}=s_{i j} a$. But, from (2.8(ii)), $s_{p j}=\phi_{j}\left(s_{p j}\right)=\phi_{j}\left(s_{i j} a\right)$, that is, $\pi_{j}(a)$ takes $i$ into $p$. Hence each block is contained in some transitivity class. Now let $p$ be some other index in the transitivity class containing $i$; then there exists an $a \in A_{j}$ such that $i \pi_{j}(a)=p$, that is, $s_{p j}=\phi_{j}\left(s_{i j} a\right)$. But, from (2.8(iii)), $\phi_{j}\left(s_{i j} a\right)=s_{i j} a^{\prime}$ for some $a^{\prime} \in A_{j}$, and so $p$ lies in the same block as $i$.

We now come to the main theorem in this section.

(4.7) TheOREM. Let S be a uniform Schreier system in $G$. Then there is a set $\Phi$ of admissible functions for $S$ if and only if to each $j \in J$, and to each block $I_{r j}$ of $I$, there is a permutation representation $\Pi_{r}\left(A_{j}\right)$ of $A_{j}$ satisfying the following conditions.

(4.8) $\Pi_{r}\left(A_{j}\right)$ is transitive on $I_{r j}$.

(4.9) If $s_{i j}=s_{p j}$ a for some $a \in A_{j}$ and for some $i, p \in I_{r j}$, then $p \pi_{r j}(a)=i$, where

$$
\pi_{r j}(a) \in \Pi_{r}\left(A_{j}\right)
$$

Proof. 1. We have already seen $(4.3,4.5)$, that, if $\Phi$ is a set of admissible functions for $S$, then we can construct permutation representations $\Pi_{r}\left(A_{j}\right)$ satisfying (4.8). (4.9) follows from the fact that, if $s_{i j}=s_{p j} a$, then $\phi_{j}\left(s_{i j}\right)=\phi_{j}\left(s_{p j} a\right)$.

2. We now assume that we are given a uniform Schreier system $S$ and permutation representations satisfying (4.8) and (4.9). We use these to define a set of admissible functions $\Phi$ for $S$ by constructing, for each $j \in J$, a function $\phi_{j}$ satisfying (2.8). We do this as follows.

(4.10) If $s_{i k}$ is in $S$ but not in $S_{j}$, we define $\phi_{j}\left(s_{i k}\right)=s_{i j}$. If $s_{i j} \in S_{j}$, for each $a \in A_{j}$, we define $\phi_{j}\left(s_{i j} a\right)=s_{p j}$, where $i \pi_{r j}(a)=p$.

We have to show that these functions $\phi_{j}$ satisfy (2.8).

(i) $\phi_{j}$ is defined on $\tilde{S}_{j}=S \cup S_{j} A_{j}$, with its values in $S_{j}$, and is clearly well defined.

(ii) Let $s_{p q} \in S$; then, if $s_{p q} \notin S_{j}, \phi_{j}\left(s_{p q}\right)=s_{p j}$. On the other hand, if $s_{p q} \in S_{j}, \pi_{r j}(1)$ fixes $p$, so that, from (4.10), $\phi_{j}\left(s_{p q} .1\right)=s_{p q}$. Hence, in either case, $\phi_{j}\left(s_{p q}\right)=s_{p j}$.

(iii) Let $s_{i j} \in S_{j}$ and $a \in A_{j}$; then $\pi_{r j}(a)$ takes $i$ into some index $p$ in the same block as $i$. That is, there exists an $s_{i_{r} j} \in S_{j}^{*}$ such that $s_{i j}=s_{i_{r} j} a^{\prime}$ and $s_{p j}=s_{i_{r} j} a^{\prime \prime}$, where $a^{\prime}, a^{\prime \prime} \in A_{j}$. Thus, from (4.10), $\phi_{j}\left(s_{i j} a\right)=s_{p j}=s_{i_{r}} a^{\prime \prime}=s_{i_{r} j} a^{\prime}\left(a^{\prime}\right)^{-1} a^{\prime \prime}=s_{i j}\left(a^{\prime}\right)^{-1} a^{\prime \prime}$.

Hence $\phi_{j}\left(s_{i j} a\right) \in s_{i j} A_{j}$.

(iv) Let $s_{i j} \in S_{j}$ and $a, a^{\prime} \in A_{j}$, and let $\phi_{j}\left(s_{i j} a\right)=s_{p j}$ and $\phi_{j}\left(s_{p j} a^{\prime}\right)=s_{q j}$. Then, from (4.10), $i \pi_{r j}(a)=p$ and $p \pi_{r j}\left(a^{\prime}\right)=q$. But $\Pi_{r}\left(A_{j}\right)$ is a permutation representation; so

$$
\pi_{r j}(a) \pi_{r j}\left(a^{\prime}\right)=\pi_{r j}\left(a a^{\prime}\right)
$$

that is, $i \pi_{r j}\left(a a^{\prime}\right)=q$, and so $\phi_{j}\left(s_{i j} a a^{\prime}\right)=s_{q j}$. That is,

$$
\phi_{j}\left(\phi_{j}\left(s_{i j} a\right) a^{\prime}\right)=\phi_{j}\left(s_{i j} a a^{\prime}\right) .
$$


Hence $\phi_{j}$ defined by (4.10) satisfies (2.8(i), (ii), (iii), (iv)), which implies that (2.8(v)) is also satisfied. Now put $\Phi=\left\{\phi_{j}\right.$, for all $\left.j \in J\right\}$; then, from (2.9), $\Phi$ is a set of admissible functions for $S$. This completes the proof.

We note that (4.8) is in fact implied by (4.9) and could therefore have been omitted.

This theorem solves problem (2.14) in that, to each of the possible sets of permutation representations $\left\{\Pi\left(A_{j}\right), j \in J\right\}$ satisfying (4.7), we obtain a set of admissible functions $\Phi$ for $S$, and every set of admissible functions for $S$ gives rise to such a set of permutation representations $\left\{\left(\Pi A_{j}\right), j \in J\right\}$, satisfying (4.7).

5. Problem (2.16). In this section, we consider problem (2.16), namely, given a subgroup $H$ of $G$, determine all its representations $H=\langle S, \Phi\rangle$. We prove the following theorem.

(5.1) Theorem. Let $G=\prod^{*} A_{j}(j \in J)$, and let $H_{1}, H_{2}$ be subgroups of $G$ such that $H_{1}=\langle S, \Phi\rangle$ and $H_{2}=\left\langle S^{\prime}, \Phi^{\prime}\right\rangle$. Then $H_{1}=H_{2}$ if and only if there exist 1-1 mappings $\mu_{j}$ of each $S_{j}$ onto $S_{j}^{\prime}(j \in J)$, such that

(i) $s_{1 j} \mu_{j}=s_{1 j}^{\prime}$,

(ii) $\phi_{j}\left(s_{1 j} a\right) \mu_{j}=\phi_{j}^{\prime}\left(s_{i j} \mu_{j} a\right)$ for all $a \in A_{j}$,

(iii) $\phi_{k}\left(s_{i j}\right) \mu_{k}=\phi_{k}^{\prime}\left(s_{i j} \mu_{j}\right)$.

Proof. 1. We first assume that $H_{1}=H_{2}$ and construct the mappings $\mu_{j}$. As in $\S 2$, let $\sim$ be the coset equivalence, so that, if $g, g^{\prime} \in G$, then $g \sim g^{\prime}$ if and only if $g$ and $g^{\prime}$ lie in the same left coset of $G$ modulo $H_{1}$. Clearly, to each $s_{i j} \in S_{j}$ there corresponds a unique $s_{p j}^{\prime} \in S_{j}^{\prime}$ such that $s_{i j} \sim s_{p j}^{\prime}$. We use this to define $\mu_{j}$ from $S_{j}$ to $S_{j}^{\prime}$ by the rule, $s_{i j} \mu_{j}=s_{p j}^{\prime}$ if and only if $s_{i j} \sim s_{p j}^{\prime}$. We have to show that the conditions (i), (ii), (iii) of the theorem are satisfied.

(i) Since $s_{1 j}=1=s_{1 j}^{\prime}, s_{1 j} \sim s_{1 j}^{\prime}$, and so $s_{1 j} \mu_{j}=s_{1 j}^{\prime}$.

(ii) Let $s_{i j} \in S_{j}$, and let $a \in A_{j}$; then, from the definition, $s_{i j} \sim s_{i j} \mu_{j}$; so $s_{i j} a \sim s_{i j} \mu_{j} a$. But, from the definition of $\phi_{j}$ and $\phi_{j}^{\prime}(2.1,2.5), \phi_{j}\left(s_{i j} a\right) \sim s_{i j} a$ and $\phi_{j}^{\prime}\left(s_{i j} \mu_{j} a\right) \sim s_{i j} \mu_{j} a$. However $\sim$ is an equivalence relation; so $\phi_{j}\left(s_{i j} a\right) \sim \phi_{j}^{\prime}\left(s_{i j} \mu_{j} a\right)$, and, from the definition of $\mu_{j}$, this implies that

$$
\phi_{j}\left(s_{i j} a\right) \mu_{j}=\phi_{j}^{\prime}\left(s_{i j} \mu_{j} a\right) \text {. }
$$

(iii) Again, suppose that $s_{i j} \in S_{j}$; then $s_{i j} \sim s_{i j} \mu_{j}$. From the definition of $\phi_{k}$ and $\phi_{k}^{\prime}$,

$$
\phi_{k}\left(s_{i j}\right) \sim s_{i j} \sim s_{i j} \mu_{j} \sim \phi_{k}^{\prime}\left(s_{i j} \mu_{j}\right),
$$

and, from the definition of the $\mu_{j}, \phi_{k}\left(s_{i j}\right) \mu_{k}=\phi_{k}^{\prime}\left(s_{i j} \mu_{j}\right)$.

2. We now assume that we are given the two subgroups

$$
H_{1}=\langle S, \Phi\rangle \text { and } H_{2}=\left\langle S^{\prime}, \Phi^{\prime}\right\rangle \text {, }
$$

and the mappings $\mu_{j}$ for all $j \in J$, satisfying the conditions (i), (ii), (iii), and we show that $H_{1}=H_{2}$. We do this by showing that the cosets $H_{1} s_{i j}$ and $H_{2} s_{i j} \mu_{j}$ are equal. We proceed by induction on the length of the elements of $G$. 
(I) If the length of $g, \lambda(g)=0$, then $g=1$ and

$$
g \in H_{1} s_{1 j} \cap H_{2} s_{1 j}^{\prime}
$$

But, from condition (i), $s_{1 j}^{\prime}=s_{1 j} \mu_{j}$; so $g \in H_{1} s_{1 j} \cap H_{2} s_{1 j} \mu_{j}$.

(II) Now let $g$ be of length $t$ and let $g=g^{\prime} a_{t}$, where $g^{\prime}$ is of length $t-1$. Then, by hypothesis, $g^{\prime} \in H_{1} s_{i j} \cap H_{2} s_{i j} \mu_{j}$ for some $s_{i j} \in S_{j}$. Now let $\psi_{j}, \psi_{j}^{\prime}$ be the extensions (3.1) of $\phi_{j}, \phi_{j}^{\prime}$ to the whole of $G$; then $s_{i j}=\psi_{j}\left(g^{\prime}\right)$ and $s_{i j} \mu_{j}=\psi_{j}^{\prime}\left(g^{\prime}\right)$. Clearly $g \in H_{1} \psi_{j}(g) \cap H_{2} \psi_{j}(g)$; so we only have to show that $\psi_{j}(g) \mu_{j}=\psi_{j}^{\prime}(g)$.

Suppose then that $a_{t} \in A_{k}$; then, from (3.5),

$$
\psi_{j}(g) \mu_{j}=\psi_{j}\left(g^{\prime} a_{t}\right) \mu_{j}=\psi_{j}\left(\psi_{j}\left(g^{\prime}\right) a_{\imath}\right) \mu_{j}=\psi_{j}\left(s_{i j} a_{\imath}\right) \mu_{j}=\phi_{j}\left(\phi_{k}\left(s_{i k} a_{t}\right)\right) \mu_{j}
$$

from (3.1).

Using condition (iii), we have

$$
\psi_{j}(g) \mu_{j}=\phi_{j}^{\prime}\left(\phi_{k}\left(s_{i k} a_{t}\right) \mu_{k}\right),
$$

which from condition (ii) implies that

$$
\psi_{j}(g) \mu_{j}=\phi_{j}^{\prime}\left(\phi_{k}^{\prime}\left(s_{i k} \mu_{k} a_{t}\right)\right)
$$

Hence, from (2.8(ii)), it follows that

$$
\psi_{j}(g) \mu_{j}=\phi_{j}^{\prime}\left(\phi_{k}^{\prime}\left(\phi_{k}^{\prime}\left(\phi_{j}^{\prime}\left(s_{i k} \mu_{k}\right)\right) a_{t}\right)\right)
$$

which, from condition (iii), is

$$
\psi_{j}(g) \mu_{j}=\phi_{j}^{\prime}\left(\phi_{k}^{\prime}\left(\phi_{k}^{\prime}\left(\phi_{j}\left(s_{i k}\right) \mu_{j}\right) a_{\mathrm{f}}\right)\right)=\phi_{j}^{\prime}\left(\phi_{k}^{\prime}\left(\phi_{k}^{\prime}\left(s_{i j} \mu_{j}\right) a_{t}\right)\right) .
$$

But the right-hand side is now $\psi_{j}\left(s_{i j} \mu_{j} a_{t}\right)$, by (3.1); so

$$
\psi_{j}(g) \mu_{j}=\psi_{j}^{\prime}\left(s_{i j} \mu_{j} a_{t}\right)=\psi_{j}^{\prime}\left(\psi_{j}^{\prime}\left(g^{\prime}\right) a_{t}\right)=\psi_{j}^{\prime}\left(g^{\prime} a_{t}\right)=\psi_{j}^{\prime}(g),
$$

and so $g \in H_{1} s_{p j} \cap H_{2} s_{p j} \mu_{j}$, for some $s_{p j} \in S_{j}$.

This shows that, for each $s_{i j} \in S_{j}$,

and in particular

$$
H_{1} s_{i j}=H_{2} s_{i j} \mu_{j}
$$

that is, $H_{1}=H_{2}$.

$$
H_{1} s_{1 j}=H_{2} s_{1 j} \mu_{j}=H_{2} s_{1 j}^{\prime}
$$

It is clear that any permutation of the rows of a uniform Schreier system leaving the first row fixed induces mappings $\mu_{j}$ satisfying the conditions of (5.1), so that it is natural to ask how many 'different' uniform Schreier systems lead to representations of the same subgroup of $G$. There is great variation in the possibilities, since if $G=A^{*} B$, where $A, B$ are cyclic of orders 2 and 3 respectively, then the normal closure of $B$ in $G$ has only one associated uniform Schreier 
system. However, if $G$ is the free group of rank 2 generated by $a$ and $b$, and $H$ is the sub group of index 2 in $G$ freely generated by $a^{2}, b, a b a^{-1}$, then, for each integer $\alpha$,

$$
S(\alpha)=\left(\begin{array}{ll}
1 & 1 \\
a^{2 \alpha+1} & a^{2 \alpha+1}
\end{array}\right)
$$

is a uniform Schreier system for $H$ in $G$.

6. Subgroup representations. We have seen that, if $H$ is a subgroup of $G$, then there are a uniform Schreier system $S$ and a set of admissible functions $\Phi$ for $S$ associated with $H$ which together determine the structure of $H$. We have also associated certain permutation representations of the free factors $A_{i}$ of $G$ with $S$ and $\Phi$, and in this section we consider connections between these representations and the free factors $s_{i j} A_{j} s_{i j}^{-1} \cap H$ of $H$.

(6.1) THEOREM. Let $s_{i j} \in S_{j}^{*}$ and let $i \in I_{r j}$, a block of $I$. Let $\Pi_{r}\left(A_{j}\right)$ be the transitive permutation representation of $A_{j}$ induced on $I_{r j}$ by $\phi_{j} \in \Phi$, and let $B_{r j}$ be the subgroup of $A_{j}$ such that

$$
s_{i j} B_{r j} s_{i j}^{-1}=s_{i j} A_{j} s_{i j}^{-1} \cap H .
$$

Then $\Pi_{r}\left(B_{r j}\right)$ is the stabilizer of $i$ in $\Pi_{r}\left(A_{j}\right)$.

Proof. 1. Let $x=s_{i j} a \phi_{j}\left(s_{i j} a\right)^{-1}$ be a typical generator of $s_{i j} B_{r j} s_{i j}^{-1}$; then, from (2.8(iii)), $\phi_{j}\left(s_{i j} a\right)=s_{i j} a^{\prime}$ for some $a^{\prime} \in A_{j}$. Hence $x=s_{i j} a\left(a^{\prime}\right)^{-1} s_{i j}^{-1}$ and $a\left(a^{\prime}\right)^{-1}$ is a generator of $B_{r j}$. We calculate $\phi_{j}\left(s_{i j} a\left(a^{\prime}\right)^{-1}\right)$, using (2.8(iv)).

$$
\phi_{j}\left(s_{i j} a\left(a^{\prime}\right)^{-1}\right)=\phi_{j}\left(\phi_{j}\left(s_{i j} a\right)\left(a^{\prime}\right)^{-1}\right)=\phi_{j}\left(s_{i j} a^{\prime}\left(a^{\prime}\right)^{-1}\right)=\phi_{j}\left(s_{i j}\right)=s_{i j} .
$$

So, in $\Pi_{r}\left(A_{j}\right), \pi_{r j}\left(a\left(a^{\prime}\right)^{-1}\right)$ fixes $i$. Similar results hold for all the generators of $B_{r j}$, and hence $\Pi_{r}\left(B_{r j}\right)$ lies in the stabilizer of $i$.

2. Now let $\pi_{\mathrm{r} j}(a)$ fix $i$, for some $a \in A_{j}$; then $\phi_{j}\left(s_{i j} a\right)=s_{i j}$ and so

that is, $a \in B_{r j}$.

$$
s_{i j} a \phi_{j}\left(s_{i j} a\right)^{-1}=s_{i j} a s_{i j}^{-1} \in s_{i j} B_{r j} s_{i j}^{-1},
$$

We now consider further the role of these $B_{r j}$, but first we introduce some more notation. Let $I_{r j}$ be a block of $I$ and $i \in I_{r j}$ for some $s_{i j} \in S^{*}$; then we denote by $A_{r j}$ the subset of $A_{j}$ such that $a \in A_{r j}$ if and only if $s_{i j} a \in S_{j}$. In particular $1 \in A_{r j}$. Clearly $\pi_{r j}(a)$, for $a \in A_{r j} a \neq 1$, does not fix $i$, and, in fact, for each $p \in I_{r j}$ there is an $a \in A_{r j}$ such that $i \pi_{r j}(a)=p$. We now recall that, if $M$ is any group and $\Pi(M)$ a permutation representation of $M$ which is transitive on a set $R$, then there is a subgroup $N$ of $M$, fixing some symbol in $R$, such that $\Pi(M)$ is the representation of $M$ induced by the left cosets of $N$ in $M$ and such that the index of $N$ in $M$ is the cardinal of the set $R$.

Applying this to our situation we see that, from (6.1), $\Pi_{r}\left(A_{j}\right)$ is the transitive permutation representation of $A_{j}$ induced by the left cosets of $B_{r j}$ in $A_{j}$, and that the index of $B_{r j}$ in $A_{j}$ is the cardinal of the set $I_{r j}$. In fact, $A_{r j}$ is a left transversal for $B_{r j}$ in $A_{j}$.

We can now distinguish between the different subgroups obtained from the various sets of admissible functions defined on a given uniform Schreier system $S$. 
(6.2) THEOREM. Let $\Phi, \Phi^{\prime}$ be two sets of admissible functions defined on a given uniform Schreier system $S$, and let $H, H^{\prime}$ be the two subgroups, $H=\langle S, \Phi\rangle, H^{\prime}=\left\langle S, \Phi^{\prime}\right\rangle$, with free decompositions

$$
\begin{array}{r}
H=F^{*} \prod_{j \in J}^{*}\left(\prod_{s_{i j} \in S_{j}^{*}}^{*} s_{i j} B_{r j} s_{i j}^{-1}\right), \\
H^{\prime}=F^{*} \prod_{j \in J}^{*}\left(\prod_{s_{i j} \in S_{j}^{*}}^{*} s_{i j} B_{t j}^{\prime} s_{i j}^{-1}\right) .
\end{array}
$$

Then

(6.3) $F=F^{\prime}$,

and

(6.4) there is a 1-1 correspondence between the $B_{r j}$ and $B_{t j}^{\prime}$ such that, if $B_{r j}$ and $B_{t j}^{\prime}$ correspond, then

(6.5) either $B_{r j}=B_{t j}^{\prime}$ or $B_{r j}, B_{t j}^{\prime}$ are two subgroups of the same index in $A_{j}$ with the same left transversal $A_{r j}$ in $A_{j}$.

Proof. From (2.11), the free factor of a subgroup associated with a uniform Schreier system $S$ is generated by the set $Q=\left\{s_{i j} s_{i q}^{-1}\right.$, for all $i \in I$ and all $\left.j, q \in J\right\}$, which depends only on $S$ and not on $\Phi$ or $\Phi^{\prime}$. Hence $F, F^{\prime}$ are generated by the same set $Q$ and so $F=F^{\prime}$.

Again, the number of $B_{r j}$ depends only on the number of blocks $I_{r j}$ or $I$, and not on $\Phi$ or $\Phi^{\prime}$, and so the $B_{r j}, B_{t j}^{\prime}$ can be put into 1-1 correspondence by the rule that $B_{r j}, B_{r j}^{\prime}$ correspond if they refer to the same block $I_{r j}$ of $I$. That is, $s_{i j} B_{r j} s_{i j}^{-1}$ is a factor of $H$ and $s_{i j} B_{r j}^{\prime} s_{i j}^{-1}$ is a factor of $H^{\prime}$ for the same $s_{i j} \in S^{*}$. Now let $\Pi_{r}\left(A_{j}\right)$ be the representation of $A_{j}$ induced by $\phi_{j} \in \Phi$ on $I_{r j}$, and $\Pi_{r}^{\prime}\left(A_{j}\right)$ be the representation of $A_{j}$ induced by $\phi_{j}^{\prime} \in \Phi^{\prime}$ also on $I_{r j}$; then we have seen that $\Pi_{r}\left(A_{j}\right)$ is induced by the left cosets of $B_{r j}$ in $A_{j}$ and $\Pi_{r}^{\prime}\left(A_{j}\right)$ is induced by the left cosets of $B_{r j}^{\prime}$ in $A_{j}$. So either $B_{r j}=B_{r j}^{\prime}$ or $B_{r j} \neq B_{r j}^{\prime}$ and both subgroups have the same index, the cardinal of $I_{r j}$, and the same left transversal $A_{r j}$ in $A_{j}$.

Again, let $H$ be any subgroup of $G$ and $H=\langle S, \Phi\rangle$ and consider the permutation representations of the free factors of $G$ induced by $S$ and $\Phi$. These representations induce a transitive permutation representation of $G$ which is essentially the representation of $G$ induced by the left cosets of $H$ in $G$. Conversely, any transitive permutation representation of $G$ is induced by the left cosets of some subgroup $H$ of $G$; however, we put this more precisely:

(6.6) TheOREM. Let $\Pi(G)$ be a transitive permutation representation of $G$ on a set $I=\{1, \ldots\}$. Then there is a uniform Schreier system $S$, and a set of admissible functions $\Phi$ for $S$, such that the following conditions hold.

(6.7) The rows of $S$ are indexed by $I$.

(6.8) $\Pi(G)$ is the representation of $G$ induced by the left cosets of the subgroup $H=\langle S, \Phi\rangle$ in $G$.

(6.9) If $s_{i j}, s_{p j} \in S_{j}$ and $a \in A_{j}$, then $\phi_{j}\left(s_{i j} a\right)=s_{p j}$ if and only if $i \pi(a)=p$. 
Proof. Let $\mu$ be the homomorphism of $G$ onto $\Pi(G), R$ be the stabilizer of 1 in $\Pi(G)$, and $H$ be the subgroup such that $H \mu=R$. Since $H$ is a subgroup of the free product $G$, there is a uniform Schreier system $S$ and a set of admissible functions for $S$ such that $H=\langle S, \Phi\rangle$. We have to show that the conditions (6.7), (6.8), (6.9) are satisfied.

First, consider the left coset $R \pi(g)$ of $R$ in $\Pi(G)$. If $\pi(g) \notin R$, there is some index $i \in I$ such that $1 \pi(g)=i$, and hence every element of $R \pi(g)$ takes 1 into $i$. Now, since $\Pi(G)$ is transitive on the set $I$, to each $i \in I$ there corresponds a $\pi(g) \in \Pi(G)$ such that $1 \pi(g)=i$, and for this $\pi(g)$ the coset $R \pi(g)$ takes 1 into $i$. That is, the left cosets of $R$ in $\Pi(G)$ can be indexed by $I$.

Now let $g \in G$; then, since $\mu$ is a homomorphism, $(H g) \mu=H \mu g \mu=R g \mu$, and each left coset of $H$ in $G$ is mapped onto some left coset of $R$ in $(\Pi G)$. Now $\mu$ is onto $\Pi(G)$ and so at least one coset, $H g$, of $H$ in $G$ is mapped onto each coset $R \pi(g)$, of $R$ in $\Pi(G)$. Suppose that $H g_{1}, H g_{2}$ are two left cosets of $H$ in $G$ such that $R \pi\left(g_{1}\right)=R \pi\left(g_{2}\right)$. Then $\pi\left(g_{1}\right)$ and $\pi\left(g_{2}\right)$ both take 1 into $i$, say, and so $\pi\left(g_{2}^{-1}\right)$ takes $i$ into 1 . That is, $\pi\left(g_{1}\right) \pi\left(g_{2}^{-1}\right)=\pi\left(g_{1} g_{2}^{-1}\right)$ fixes 1 . But from the definition of $H$, this implies that $g_{1} g_{2}^{-1} \in H$, and so $H g_{1}=H_{2}$. Hence at most one left coset of $H$ in $G$ is mapped onto each left coset of $R$ in $\Pi(G)$. That is, there is a 1-1 correspondence between the left cosets of $H$ in $G$ and the left cosets of $R$ in $\Pi(G)$, which we have seen can be indexed by $I$. Now the rows of $S$ correspond to the left cosets of $H$ in $G$ and so can also be indexed by $I$. In particular the first row of $S$ is indexed by 1 , since it corresponds to the coset $H$. This proves (6.7).

Now let $s_{i j}, s_{p j} \in S_{j}$; then $\pi\left(s_{i j}\right)$ takes 1 into $i$, and $\pi\left(s_{p j}\right)$ takes 1 into $p$. Suppose that $\phi_{j}\left(s_{i j} a\right)=s_{p j}$ for some $a \in A_{j}$; then this implies that $H s_{i j} a=H s_{p j}$, and so $\left(s_{i j} a\right) \mu=s_{p j}$. That is, $\pi\left(s_{i j}\right) \pi(a)=\pi\left(s_{p j}\right)$, and so $i \pi(a)=p$. Now let $a \in A_{j}$ be such that $i \pi(a)=p$; then $\pi\left(s_{i j}\right) \pi(a)=\pi\left(s_{p j}\right)$, and so $\left(s_{i j} a\right) \mu=s_{p j} \mu$. This implies that $H s_{i j} a=H s_{p j}$, and so, from the definition of $\phi_{j}, \phi_{j}\left(s_{i j} a\right)=s_{p j}$, which proves (6.9). This also proves (6.8), since $\Pi(G)$ is then the permutation representation of $G$ induced by the left cosets of $H$ in $G$, which has the given representation $H=\langle S, \Phi\rangle$.

We now come to the theorem on subgroups of finite index in $G$.

(6.10) Theorem. Let $G=\prod^{*} A_{j}(j \in J)$, where $J$ is the finite set $\{1,2, \ldots, k\}$, and let $d_{j}^{n}(n>0)$ be the number of homomorphisms of $A_{j}$ into the symmetric group on $n$ symbols and $d_{j}^{0}=1$. Let $N_{n}$ be the number of subgroups of $G$ of index $n$; then $N_{1}=1$ and, if $n>1$,

$$
N_{n}=\{1 /(n-1) !\} \prod_{j=1}^{k} d_{j}^{n}-\sum_{i=1}^{n-1}\{1 /(n-i) !\} \prod_{j=1}^{k} d_{j}^{n-i} N_{i}
$$

Proof. (i) $N_{1}=1$ states that $G$ is its own unique subgroup of index 1 .

(ii) The integer $d_{j}^{n}$ is the number of permutation representations of $A_{j}$ on $n$ symbols. If, for each $j \in J$, we take a permutation representation $\Pi\left(A_{j}\right)$ of $A_{j}$ and put

$$
\Pi(G)=\operatorname{gp}\left\{\Pi\left(A_{j}\right): \quad j \in J\right\},
$$

we obtain a permutation representation of $G$ on $n$ symbols. Therefore the total number of permutation representations of $G$ on $n$ symbols is $\prod_{j=1}^{k} d_{j}^{n}$. 
Now let $\Pi(G)$ be a particular permutation representation of $G$ on $n$ symbols, let

$$
\left\{1, b_{2}, b_{3}, \ldots, b_{i}\right\}
$$

be the transitivity class containing 1 , and let $\Pi^{i}(G)$ be the transitive permutation representation of $G$ induced by $\Pi(G)$ on these $i$ symbols. Then, from (6.6), there is a unique subgroup $H$ of index $i$ in $G$ corresponding to $\Pi^{i}(G)$. We now consider the total number of permutation representations of $G$ on $n$ symbols which give rise to this subgroup $H$. Clearly, it does not matter what permutation representation of $G$ on the remaining $n-i$ symbols is taken. Also, since any permutation of the symbols $b_{2}, b_{3}, \ldots b_{i}$ gives rise to the same subgroup $H$, with each subgroup of index $i$ in $G$ we can associate

$$
(n-1) \ldots(n-i+1) \prod_{j=1}^{k} d_{j}^{n-i}
$$

permutation representations of $G$ on $n$ symbols. Hence

$$
\prod_{j=1}^{k} d_{j}^{n}=\sum_{i=1}^{n}\{(n-1) ! /(n-i) !\} \prod_{j=1}^{k} d_{j}^{n-i} N_{i}
$$

and on rearranging we get

$$
N_{n}=\{1 /(n-1) !\} \prod_{j=1}^{k} d_{j}^{n}-\sum_{i=1}^{n-1}\{1 /(n-i) !\} \prod_{j=1}^{k} d_{j}^{n-i} N_{i} .
$$

As an example, consider the case where each $A_{j}$ is an infinite cyclic group; then $G$ is a free group of finite rank $k$. Since the generator of $A_{j}$ can be mapped onto any element in the symmetric group on $n$ symbols, $d_{j}^{n}=n$ ! Hence

$$
\begin{aligned}
N_{n} & =\{1 /(n-1) !\}(n !)^{k}-\sum_{i=1}^{n-1}\{1 /(n-i) !\}\{(n-i) !\}^{k} N_{i}, \\
& =n(n !)^{k-1}-\sum_{i=1}^{n-1}((n-i) !)^{k-1} N_{i},
\end{aligned}
$$

which is the formula of Hall [3].

Let us now consider normal subgroups of $G$, and investigate their effect on uniform Schreier systems and on the permutation representations $\Pi(G)$.

We start by proving the following theorem.

(6.12) TheOrem. Let $G=\prod^{*} A_{j}(j \in J)$, and let $H$ be a subgroup of $G$. Let $S$ be a uniform Schreier system and $\Phi$ a set of admissible functions for $S$, such that $H=\langle S, \Phi\rangle$. Let $\psi_{1}$ be the extension of $\phi_{1}$ in the sense of (3.1) and let $B=\bigcup_{j \in J} A_{j}$. Then $H$ is normal in $G$ if and only if, for all $b \in B$,

(i) $\psi_{1}\left(b s_{i j} a\right)=\psi_{1}\left(b \phi_{j}\left(s_{i j} a\right)\right)$, for all $s_{i j} a \in S_{j} A_{j}$ and all $j \in J$,

(ii) $\psi_{1}\left(b s_{i j}\right)=\psi_{1}\left(b s_{i q}\right)$, for all $j, q \in J$ and all $i \in I$.

Proof. 1. First, let $H$ be normal in $G$; then, from (2.11) and (2.12), $H$ is generated by elements of the form $s_{i j} s_{i q}^{-1}$ and $s_{i j} a \phi_{j}\left(s_{i j} a\right)^{-1}$. Now let $b \in B$; then, since $H$ is normal in $G$,

$$
b s_{i j} s_{i q}^{-1} b^{-1} \in H \text { and } b s_{i j} a \phi_{j}\left(s_{i j} a\right)^{-1} b^{-1} \in H \text {. }
$$


This implies that $b s_{i j}$ and $b s_{i q}$ lie in the same coset of $H$, and that $b s_{i j} a$ and $b \phi_{j}\left(s_{i j} a\right)$ also lie in the same coset of $H$. Hence, from the properties of $\psi_{1}$,

and

$$
\psi_{1}\left(b s_{i j}\right)=\psi_{1}\left(b s_{i q}\right)
$$

$$
\psi_{1}\left(b s_{i j} a\right)=\psi_{1}\left(b \phi_{j}\left(s_{i j} a\right)\right) .
$$

2. Now let $H$ be a subgroup of $G$ and let $H=\langle S, \Phi\rangle$, where $S$, $\Phi$ satisfy the statement of the theorem. That is,

and

$$
\psi_{1}\left(b s_{i j} a\right)=\psi_{1}\left(b \phi_{j}\left(s_{i j} a\right)\right)
$$

This implies that

$$
\psi_{1}\left(b s_{i j}\right)=\psi_{1}\left(b s_{i q}\right) .
$$

$$
b s_{i j} s_{i q}^{-1} b^{-1} \in H \text { and } b s_{i j} a \phi_{j}\left(s_{i j} a\right)^{-1} b^{-1} \in H .
$$

Now $s_{i j} a \phi_{j}\left(s_{i j} a\right)^{-1}$ and $s_{i j} s_{i q}^{-1}$ run over all the generators of $H$, so that, by the obvious extension to $H, b H b^{-1}=H$, for all $b \in B$. Now $B=\bigcup_{j J} A_{j}$, and so, again by extending to the elements of $G$ in an obvious way, $g^{-1} H g=H$, for all $g \in G$. That is, $H$ is normal in $G$.

Again suppose that the $H$ normal in $G$ has the free decomposition

$$
H=F^{*} \prod_{j \in J}^{*}\left(\prod_{s_{i j} \in S_{j}}^{*} s_{i j} A_{j} s_{i j}^{-1} \cap H\right)
$$

and, as in (6.1), put $s_{i j} B_{r j} s_{i j}^{-1}=s_{i j} A_{j} s_{i j}^{-1} \cap H$, where $i \in I_{r j}$, a block of $I$; then it is easy to show that $B_{r j}=A_{j} \cap H$ for all $r$ and that $B_{r j}$ is normal in $A_{j}$. Applying this observation to the earlier results in this section we find that the cardinal of each block $I_{r j}$ is the same for all $r$, being the index of $A_{j} \cap H$ in $A_{j}$, and that $\Pi_{r}\left(A_{j}\right)$ is the same representation for each $r$, being that representation of $A_{j}$ induced by the cosets of $A_{j} \cap H$ in $A_{j}$.

As a simple consequence of these facts, if we consider a group $G=A * B$, where $A, B$ are cyclic of prime order, then the only normal subgroups of $G$, apart from the normal closures of $A$ and $B$ in $G$, are free subgroups, that is when $\Pi_{r}(A), \Pi_{r}(B)$ are regular representations of $A$ and $B$. In such a situation a formula analogous to (6.11) can be proved which counts the number of free subgroups of finite index in $G$ in terms of those permutation representations of $G$ which lead to free subgroups (Dey $[1$, p. 71$]$ ).

7. Residual finiteness. It is well known that the free product of two residually finite groups is residually finite (Gruenberg [2]); however, to illustrate the methods evolved in this paper we give a simple proof of this theorem.

(7.1) TheOREM. Let $G=A_{1} * A_{2}$ be the free product of two residually finite groups $A_{1}, A_{2}$; then $G$ is residually finite.

Proof. Let $g \in G, g \neq 1$; then we have to find a normal subgroup $N$ of finite index in $G$ such that $g \notin N$. Since $g \in G, g$ has a unique representation as a reduced word in syllables from $A_{1}$ and $A_{2}, g=a_{1} a_{2} \ldots a_{t}$ such that no two adjacent syllables lie in the same $A_{j}(j \in J=\{1,2\})$. 
Put $I=\{1,2, \ldots, t, t+1\}$ and construct the following subset $S^{\prime}$ of $G: S^{\prime}=\left\{s_{i j}^{\prime}: i \in I, j \in J\right\}$, where, for all $j \in J$,

(i) $s_{1 j}^{\prime}=1$,

(ii) $s_{i j}^{\prime}=a_{1} a_{2} \ldots a_{i-1} \quad(i \neq 1)$.

Clearly $S^{\prime}$ is a uniform Schreier system in $G$ such that each block $I_{r j}(4.5)$ contains either one or two indices and there are only finitely many such blocks. Now, from (4.7), there is a set of admissible functions $\Phi^{\prime}$ for $S^{\prime}$ if for each block $I_{r j}$ there is a transitive permutation representation $\Pi_{r}\left(A_{j}\right)$ of $A_{j}$ on $I_{r j}$. Consider any block $I_{r j}$; then either there is such a representation or there is not and if not, then the block $I_{r j}$ must contain two indices. Suppose that there is no such representation on the block $I_{r j}=\left\{i_{1}, i_{2}\right\}$; then $s_{i_{1 j}}=s, s_{i_{2} j}=s a$, where $a \in A_{j}$, $s \in S^{\prime}$ and the last syllable of $s$ does not lie in $A_{j}$. Since $A_{j}$ is residually finite, we can choose a transversal $1, a, a^{\prime}, a^{\prime \prime}, \ldots, a^{(n)}$ for a normal subgroup of finite index in $A_{j}$ which does not contain the element $a$. Now, putting

$$
I=\{1,2, \ldots, t+1, t+2, \ldots, t+n+1\},
$$

we form the subset $S^{\prime \prime}$ of $G, S^{\prime \prime}=\left\{s_{i j}^{\prime \prime}: i \in I, j \in J\right\}$ by taking

(i) $s_{i j}^{\prime \prime}=s_{i j}^{\prime}$ for $j \in J, 1 \leqq i \leqq t+1$,

(ii) $s_{i j}^{\prime \prime}=s a^{(i-t-1)}$ for $j \in J, t+1<i \leqq t+n+1$.

That is, we have added further rows to $S^{\prime}$ in such a way that $S^{\prime \prime}$ is still a uniform Schreier system and the number of blocks $I_{r j}$ is unaltered but there is now a transitive permutation representation $\Pi_{r}\left(A_{j}\right)$ of $A_{j}$ on the block corresponding to the elements $s, s a, s a^{\prime}, \ldots, s a^{(n)}$ in $S_{j}^{\prime \prime}$.

However, if we consider blocks of the form $I_{r k}$, where $k$ is the other index in $J$ different from $j$, then there are $p$ more such blocks in $S^{\prime \prime}$ than in $S^{\prime}$. Now each of these additional blocks contains precisely one index and so the number of blocks $I_{r k}$ which do not give rise to a transitive permutation representation of $A_{k}$ is still the same. However, the number of blocks $I_{r j}$ which do not give rise to a transitive permutation representation of $A_{j}$ has been reduced by one.

We can therefore repeat this process and, by adding finitely many rows to the uniform Schreier system at each stage, we shall eventually obtain a uniform Schreier system $S$ in $G$ such that $g \in S$ and there is a transitive permutation representation $\Pi_{r}\left(A_{j}\right)$ of $A_{j}$ on each of the blocks $I_{r j}$, for each $j \in J$. Hence there is a set of admissible functions $\Phi$ for $S$ which, from (3.11), implies the existence of a subgroup $H$ of $G$ such that each column of $S$ is a left transversal for $H$ in $G$. That is, $H$ is of finite index in $G$ and $g \notin H$. Now let $N$ be the intersection of all the conjugates of $H$ in $G$ (there are only finitely many such conjugates); then $N$ is a normal subgroup of finite index in $G$ and $g \notin N$. This completes the proof.

\section{REFERENCES}

1. I. M. S. Dey, Schreier systems in free products, Ph.D. Thesis (Manchester, 1963).

2. K. W. Gruenberg, Residual properties of infinite soluble groups, Proc. London Math.'Soc. (3) 7 (1957), 29-62.

3. M. Hall, Subgroups of finite index in free groups, Canad. J. Math. 1 (1949), 187-190. 
4. M. Hall, Coset representation in free groups, Trans. Amer. Math. Soc. 67 (1949), 421-432.

5. M. Hall and T. Rado, On Schreier systems in free groups, Trans. Amer. Math. Soc. 64 (1948), 386-408.

6. H. W. Kuhn, Subgroup theorems for groups presented by generators and relations, Ann. of Math. (2) 56 (1952), 22-46.

7. S. Maclane, A proof of the Subgroup Theorem for free products, Mathematika 5 (1958), 13-19.

8. O. Schreier, Die Untergruppen der freien Gruppen, Abh. Math. Sem. Univ. Hamburg 5 (1927), 161-183.

9. A. J. Weir, The Reidemeister-Schreier and Kurosh subgroup theorems, Mathematika 3 (1956), 47-55.

UNIVERSITY OF SUSSEX 